\title{
Shed Syndecan-1 is involved in chemotherapy resistance via the EGFR pathway in colorectal cancer
}

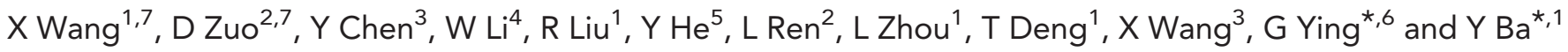

${ }^{1}$ Key Laboratory of Cancer Prevention and Therapy, Department of Gastrointestinal Oncology, National Clinical Research Center for Cancer, Tianjin Medical University Cancer Institute and Hospital, Tianjin 300060, China; ${ }^{2}$ Key Laboratory of Cancer Prevention and Therapy, Department of Clinical Laboratory, National Clinical Research Center for Cancer, Tianjin Medical University Cancer Institute and Hospital, Tianjin 300060, China; ${ }^{3}$ Guangdong Provincial Key Laboratory of Gastroenterology, Department of Digestive Diseases, Nanfang Hospital, Southern Medical University, Guangzhou 510515, China; ${ }^{4}$ Department of Cardiovascular Medicine, Tianjin Chest Hospital, Tianjin 300000, China; ${ }^{5}$ Department of Hepatology and Infectious Disease, Nanfang Hospital, Southern Medical University, Guangzhou 510515, China and 'Laboratory of Cancer Cell Biology, Key Laboratory of Cancer Prevention and Therapy, National Clinical Research Center for Cancer, Tianjin Medical University Cancer Institute and Hospital, Tianjin 300060, China
\end{abstract}

Background: Syndecan-1 (Sdc-1) shedding induced by matrix metalloproteinase-7 (MMP-7) and additional proteases has an important role in cancer development. However, the impact of Sdc-1 shedding on chemotherapeutic resistance has not been reported.

Methods: We examined Sdc-1 shedding in colorectal cancer by enzyme-linked immunosorbent assay (ELISA), Dot blot, reverse transcription-PCR (RT-PCR), immunohistochemistry and so on, its impact on chemotherapeutic sensitivity by collagen gel droplet embedded culture-drug sensitivity test (CD-DST) and MTT (3-(4,5-dimethylthiazol-2-yl)-2,5-diphenyl tetrazolium bromide), and potential mechanisms of action by Dot blot, western blot and immunofluorescence.

Results: Sdc-1 shedding was increased in colorectal cancer patients, Sdc-1 serum levels in postoperative patients were lower than in preoperative patients, but still higher than those observed in healthy adults. Patients with high preoperative Sdc-1 serum levels were less responsive to 5-Fluorouracil, Oxaliplatin, Irintecan, Cisplatin or Paclitaxel chemotherapy. Moreover, the disease-free survival of patients with high preoperative Sdc-1 serum levels was significantly poorer. The possible mechanism of chemotherapy resistance in colorectal cancer can be attributed to Sdc-1 shedding, which enhances EGFR phosphorylation and downstream signalling.

Conclusions: Shed Sdc-1 is involved in chemotherapy resistance via the EGFR pathway in colorectal cancer, and Sdc-1 serum levels could be a new prognostic marker in colorectal cancer.

Syndecan-1 (CD138, Sdc-1), a member of the heparan sulphate proteoglycan family, mainly presents on the cell membrane. It consists of a transmembrane core protein, heparan sulphate (HS) and chondroitin sulphatase chains, which are attached to the extracellular region of the transmembrane core protein (Teng et al, 2012). Since intact HS chains can bind cytokines, growth factors, chemotactic factors and extracellular matrix components, Sdc-1 has important roles in the maintenance of cell morphogenesis,

\footnotetext{
*Correspondence: Professor Y Ba; E-mail: dryiba@gmail.com or yiba99@yahoo.com or Professor G Ying; E-mail: yingguoguang@hotmail.com ${ }^{7}$ These authors contributed equally to this work.
} 
promotion of tissue repair and the regulation of immune function (Gotte, 2003). Physiologically, only a few Sdc-1 molecules are cut by sheddases, such as matrix metalloproteinase-7 (MMP-7), MMP-9, heparanase, resulting in free Sdc-1 ectodomain containing HS chains that eventually migrate to the bloodstream (Fitzgerald et al, 2000; Andrian et al, 2006; Brule et al, 2006; Yang et al, 2007).

Activated Sdc-1 shedding is observed in many pathological circumstances, including tumour cell proliferation and invasion (Nikolova et al, 2009). Many studies have confirmed that Sdc-1 shedding from the membrane of pancreatic, lung and larynx tumour cells is accelerated in comparison with shedding from the membrane of normal cells (Joensuu et al, 2002; Anttonen et al, 2003, 2006). In addition, high pretreatment Sdc-1 serum levels are associated with poor prognosis in lung cancer (small cell and nonsmall cell carcinoma) treated with platinum-based chemotherapy (Joensuu et al, 2002; Anttonen et al, 2003). Furthermore, low posttreatment Sdc-1 serum levels in larynx and hypopharynx cancer can prognosticate the sensitivity to anticancer therapy, and high posttreatment Sdc-1 serum levels are an indicator of relapse (Anttonen et al, 2006).Nevertheless, whether Sdc-1 influences the effectiveness of cancer chemotherapy remains to be elucidated.

Studies have indicated that Sdc-1 shedding is mainly caused by MMP-7, MMP-9 and heparanase also can induce Sdc-1 shedding by leading to enhanced expression of MMP-9. Intact HS chains, which are present in soluble Sdc-1, can bind to growth factors such as heparin-binding epithelial growth factor-like factor (HB-EGF), and thus can activate EGFR and downstream signalling pathways (Mythreye and Blobe, 2009). In addition, there is evidence the downstream pathways are involved in tumour drug resistance (Chen et al, 2012), especially in colorectal cancer cells (Pentheroudakis et al, 2013). Therefore, it can be speculated that soluble serum Sdc-1 may induce chemotherapy resistance via HBEGF. Most studies have mainly focused on Sdc-1 anchored on the surface of colorectal cancer cells. These studies have revealed that anchored Sdc-1 is associated with cell proliferation, transformation and metastasis in colorectal cancer (Fujiya et al, 2001; Mennerich et al, 2004; Hashimoto et al, 2008). The structure and roles of shed Sdc-1 are different from those of Sdc-1 anchored on the surface of the cell membrane (Mythreye and Blobe, 2009); however, the role of Sdc-1 shedding in colorectal tumours and their chemotherapeutic sensitivity has rarely been studied. Is Sdc-1 shedding a universal phenomenon or a particular event in cancer? Or are levels of shed Sdc-1 in colorectal cancer different from those in normal cells, and does shed Sdc-1 have an impact on chemotherapy sensitivity? Several important questions remain to be answered.

The aim of our study was to examine the change in Sdc-1 shedding in colorectal cancer and its impact on the sensitivity to chemotherapy, as well as to explore its potential mechanisms of action.

\section{MATERIALS AND METHODS}

Human tissue and serum samples. This study included 63 persons who were recruited from the Tianjin Medical University Cancer Institute and Hospital of China from February 2009 to February 2011. This group included patients with resectable primary colorectal cancers $(n=52)$, and healthy adults $(n=11)$. Each patient underwent colorectal cancer biopsy (endoscopic bite biopsy) before operation, and the final postoperative diagnosis was colorectal cancer. Patients' clinical features are shown in Table 1. Written informed consent was obtained from each patient in the study. The study was approved by the Tianjin Medical University human investigation committee, and informed consent was obtained from patients.
Cell culture. The human colonic epithelial carcinoma cell line HT-29 was obtained from the American Type Culture Collection (ATCC). Cells were cultured in humidified incubators at $37^{\circ} \mathrm{C}, 5 \% \mathrm{CO}_{2}$ in McCoy's $5 \mathrm{~A}$ medium (Sigma-Aldrich, St Louis, MO, USA) supplemented with $10 \%$ fetal bovine serum, 10 units per $\mathrm{ml}$ penicillin/streptomycin, and $10 \mu \mathrm{g} \mathrm{ml}^{-1}$ fungizone (Sigma-Aldrich).

Plasmids. cDNA encoding MMP-7, MMP-9 or heparanase was generated from human colon tissue by reverse transcription-PCR (RT-PCR) and was subcloned into a pCDH vector (BioCat, Heidelberg, Germany). Cells were transiently transfected using Lipofectamine LTX (Invitrogen, Carlsbad, CA, USA) according to the manufacturer's instructions to generate stable cell lines that permanently expressed exogenous MMP-7, MMP-9 or heparanase. Transient gene expression (TGE) cells were transfected with $1 \mu \mathrm{g}$ of linearised DNA selected for Puromycin resistance using $1 \mu \mathrm{g} \mathrm{ml}^{-1}$ of Puromycin (Sigma-Aldrich).

RNA interference. To silence the expression of human MMP-7 and HB-EGF, the following oligonucleotides (Invitrogen) were used: MMP-7 sense: $5^{\prime}$-CCGGCCTACCTATAACTGGAATGTTC TCGAGAACATTCCAGTTATAGGATGGTTTTTG- ${ }^{\prime}$ and MM P-7 antisense: $5^{\prime}$-CCGGCGTATCATATACTCGAGACTTCTCGA GAAGTCTCGAGTATATGATACGTTTTTG-3'; HB-EGF sense: 5'-CCGGAGGATTGGGCCTCCCATAATTCTCGAGAATTATG GGAGGCCCAATCCTTTTTTG-3' and HB-EGF antisense; 5'-CCGGGAGGAGGTTATGATGTGGAAACTCGAGTTTCCAC ATCATAACCTCCTCTTTTTG-3'.

As a negative control, scrambled siRNA was used. The annealed oligonucleotide duplex siRNA (10 nM) was transfected into cells using Lipofectamine RNAi max (Invitrogen) according to the manufacturer's instructions.

Antibodies. All the antibodies were obtained from commercial sources as follows: anti-MMP-7, anti-MMP-9, anti-heparanase, anti-syndecan-1 and anti-HB-EGF (Proteintech Group, Inc., Chicago, IL, USA), anti-Ras antibody (Upstate Biotechnology, Inc., Waltham, MA, USA), anti-EGFR, anti-phosphorylated-EGFR (Tyr. 1068), anti-extracellular signal-regulated kinase (ERK)1/2, anti-phosphorylated-ERK1/2 (Thr-202/Tyr-204), anti-MAPK/ERK kinase (MEK)1/2 and anti-phosphorylated-MEK1/2 (Ser-217/221) (Cell Signaling Technology Inc., Beverly, MA, USA), and antiGAPDH (Santa Cruz Biotechnology Inc., Santa Cruz, CA, USA).

Inhibitors. The inhibitors used in this study were as follows: the EGFR inhibitor AG1478, the Ras inhibitor manumycin A and ERK inhibitor FR180204 (Calbiochem, San Diego, CA, USA), the c-Raf inhibitor GW5074 and the MEK inhibitor PD98059 (Sigma-Aldrich).

Detection of Sdc-1 and MMP-7 serum levels by enzyme-linked immunosorbent assays. For the detection of Sdc-1 and MMP-7 serum levels, peripheral blood of patients was collected using pyrogen/endotoxin-free collecting tubes within 7 days preoperation and postoperation. Serum was removed rapidly and carefully from the red cells after clotting. Following clotting, the samples were centrifuged at $\sim 1000 \mathrm{~g}$ for $10 \mathrm{~min}$ and serum was removed. Samples were aliquoted $(250-500 \mu \mathrm{l})$ to avoid repeated freeze-thaw cycles and stored at $-70{ }^{\circ} \mathrm{C}$ until further use. Serum Sdc-1 levels and serum MMP-7 levels were detected by enzyme-linked immunosorbent assay (ELISA) (Diaclone, Besancon Cedex, France) as described previously (Zhang et al, 2013).

Dot blot assay analysis of MMP-7, MMP-9, heparanase and soluble Sdc-1, soluble HB-EGF, EGFR, pEGFR and GAPDH. For soluble Sdc-1, soluble HB-EGF, MMP-7 and MMP-9 detection, cell supernatant was collected. Cell lysate protein extraction was performed for heparanase, EGFR, pEGFR and GAPDH detection. 
Table 1. Clinicopathological parameters of patients with high and low Sdc-1 levels

\begin{tabular}{|c|c|c|c|c|c|}
\hline $\begin{array}{l}\text { Clinical } \\
\text { information }\end{array}$ & Total & $\begin{array}{c}\text { Sdc1 high } \\
\text { levels } \\
(N=26) \\
\text { case }(\%)\end{array}$ & $\begin{array}{c}\text { Sdc1 low } \\
\text { levels } \\
(N=26) \\
\text { case }(\%)\end{array}$ & $z$ or $\chi^{2}$ & $P$-value \\
\hline \multicolumn{6}{|l|}{ Age (years) } \\
\hline$>70$ & 10 & $5(50)$ & $5(50)$ & -0.656 & 0.512 \\
\hline 50-70 & 31 & $17(55)$ & $14(45)$ & & \\
\hline$<50$ & 11 & $4(36)$ & $7(64)$ & & \\
\hline \multicolumn{6}{|l|}{ Gender } \\
\hline Male & 25 & $12(48)$ & $13(52)$ & 0.077 & 0.781 \\
\hline Female & 27 & $14(52)$ & $13(48)$ & & \\
\hline
\end{tabular}

Stage (TNM)

\begin{tabular}{|c|c|c|c|c|c|}
\hline I & 3 & $0(0)$ & $3(100)$ & \multirow[t]{4}{*}{-2.144} & \multirow[t]{4}{*}{0.032} \\
\hline ॥ & 29 & $13(45)$ & $16(55)$ & & \\
\hline III & 12 & 7 (58) & $5(42)$ & & \\
\hline IV & 8 & $6(75)$ & $2(25)$ & & \\
\hline \multicolumn{6}{|c|}{ Tumour invasion $(\mathrm{T})$} \\
\hline T1-T3 & 7 & 1 (14) & $6(86)$ & 4.127 & 0.042 \\
\hline T4 & 45 & $25(56)$ & $20(44)$ & & \\
\hline
\end{tabular}

Number of nodes examined (N)

\begin{tabular}{|l|l|l|l|l|l|}
\hline$<12$ & 28 & $13(46)$ & $15(54)$ & 0.310 & 0.578 \\
\hline$\geqslant 12$ & 24 & $13(54)$ & $11(46)$ & & \\
\hline
\end{tabular}

Histological grade (G)

\begin{tabular}{|l|l|l|r|r|r|}
\hline $\mathrm{G} 1+\mathrm{G} 2$ & 36 & $16(44)$ & $20(56)$ & 1.444 & 0.229 \\
$\mathrm{G} 3+\mathrm{G} 4$ & 16 & $10(63)$ & $6(37)$ & & \\
\hline
\end{tabular}

\section{Lymphovascular invasion (LVI)}

\begin{tabular}{|l|r|r|r|r|r|}
\hline Present & 3 & $2(67)$ & $\begin{array}{r}1(33) \\
25(51)\end{array}$ & 0.354 & 0.552 \\
Not present & 49 & $24(49)$ & \\
\hline Incised margin (R) \\
\hline R0 & 49 & $24(49)$ & $25(51)$ & 0.354 & 0.552 \\
R1 + R2 & 3 & $2(67)$ & $1(33)$ & & \\
\hline
\end{tabular}

Colon perforation or obstruction

\begin{tabular}{|l|l|l|r|r|l|}
\hline Present & 11 & $6(55)$ & $5(45)$ & 0.115 & 0.734 \\
Not present & 41 & $20(49)$ & $21(51)$ & & \\
\hline
\end{tabular}

Postoperative adjuvant chemotherapy cycle

\begin{tabular}{|l|l|l|l|l|l|}
\hline FOLFOX $<6$ cycle & 24 & $12(50)$ & $12(50)$ & 0.000 & 1.000 \\
FOLFOX $\geqslant 6$ cycle & 28 & $14(50)$ & $14(50)$ & & \\
\hline
\end{tabular}

Abbreviations: Sdc-1 = Syndecan-1; TNM = tumour, lymph node and metastasis. Statistical analysis was performed by Pearson $\chi^{2}$ test or Mann-Whitney $U$ test. $P<0.05$ was considered as significant.

Two microliters of cell supernatant or cell lysate protein was applied to ABS-Tween moistened Immobilon-Ny+ membrane (Millipore, Billerica, MA, USA) under a mild vacuum in dotblot apparatus (Minifold, Sigma-Aldrich). The membranes were washed twice in $200 \mathrm{ml}$ of ABS-Tween for $30 \mathrm{~min}$ each time under a mild vacuum, blocked in TBS-skimmed-milk solution for $1 \mathrm{~h}$ at room temperature and incubated in TTM-primary antibody solution overnight at $4{ }^{\circ} \mathrm{C}$. The membranes were then washed twice in $60 \mathrm{ml}$ of TTM for $30 \mathrm{~min}$ each time and incubated in TTM-secondary antibody (HRP-conjugated goat anti-rat antibody, $1 \mathrm{mg} \mathrm{ml}^{-1}$ ) solution for $2 \mathrm{~h}$ at room temperature. After washing twice, dots were visualised by placing the membranes in ECL solution and then exposed. To measure the relative density of immunoreactive dots, images were scanned and analysed by the Image J Software (National Institute of Health, Bethesda, MD, USA). Dot blot analysis was performed as described previously (Wang et al, 2011). The experiments were independently performed three times.
RNA extraction and amplification by reverse transcriptionPCR. Total tissue RNA was extracted from fresh colon tissue using TRIzol reagent (Invitrogen). Reverse transcription-PCR was performed using the Advantage Two-Step RT-PCR kit (Fermentas, Hanover, MD, USA) according to the manufacturer's guidelines. The forward primer of human Sdc-1 is $5^{\prime}$-CAGCAGCAACACCG AGAC- $3^{\prime}$, and reverse primer is $3^{\prime}$-CTCCTACCTTGACGGTT $\mathrm{AG}-5^{\prime}$, which generate a product of $359 \mathrm{bp}$. The forward primer of human MMP7 is $5^{\prime}$-TTCAGACTTACCTCGGATCGTAGTGG AT- $3^{\prime}$, and reverse primer is $3^{\prime}$-GAGAAGACAAGGGCCATGAC ACTACATG $-5^{\prime}$, which generate a product of $349 \mathrm{bp}$. The relative density of Sdc-1, MMP7 hybridising bands was normalised to the amount of GAPDH product for each sample using the Image-Pro Plus 6.0 (Media Cybernetics, Inc., Rockville, MD, USA) analysis software.

Sdc-1 immunohistochemistry and semiquantitative evaluation of stain. Sections were stained by immunohistochemistry for Sdc-1 using an immunohistochemistry kit (Fuzhou Maixin Biotechnology Development Co., Ltd, Fujian, China). Negative controls included serial sections incubated without antibody, and sections incubated with non-specific rat $\operatorname{IgG}$ at an equivalent protein concentration. The images were captured by a Nikon Eclipse E600 microscope (Tokyo, Japan). Only the membranes of surface epithelial cells and glandular epithelium cells with staining were scored. The semiquantitative evaluation of stain was performed as described previously (Wang et al, 2011).

EGFR or KRAS status in HT-29 detected by real-time PCR. DNA of HT-29 cell line was extracted by QIAamp DNA Mini Kit (Qiagen, Valencia, CA, USA), EGFR or KRAS mutations were detected by therascreen real-time PCR kit (Qiagen). For each DNA sample, the control and mutation assays must be analysed on the same PCR run to avoid run-to-run variations. If there is typical S curve including index phase, linear phase, plateau phase, and CT values were less than 35 , then the sample is called mutation positive. If there is no typical S curve or CT values were more than 38 , then the sample is reported as mutation negative.

Cell surface Sdc-1 detected by immunofluorescence. Cells were seeded into 8 well-Culture Slide at $3 \times 10^{4}$ cells per well and treated as described as above. The media was removed and cells fixed in $100 \%$ cold methanol on ice for $10 \mathrm{~min}$ followed by $4 \%$ paraformaldehyde at room temperature for $2 \mathrm{~min}$. Cells were blocked with $2.5 \%$ goat serum and $2 \%$ BSA in TBS for $1 \mathrm{~h}$ at room temperature, then incubated with 1:400 primary antibodies for Syndecan-1 (Proteintech Group, Inc.) in 2\% BSA in TBST overnight at $4{ }^{\circ} \mathrm{C}$. Secondary antibodies (Alexa Fluor 568 goat anti-mouse IgG, 1:500) were applied in 2\% BSA in TBST for $1 \mathrm{~h}$ at room temperature. Cell nucleus was stained with DAPI for $5 \mathrm{~min}$. Slides were cover-slipped using Fluoromount-G and images were captured with inverted microscope (Olympus, Center Valley, PA, USA). The relative fluorescence intensity was quantified using the Image J software (NIH) and reported as relative fluorescence units (RFUs).

Western blot assay analysis of pEGFR, EGFR, Ras-GTP, Ras, p-c-Raf, p-MEK, MEK, p-ERK1/2, ERK1/2 and GAPDH. Protein extraction and western blot analysis were performed as described previously (Anegawa et al, 2008). To measure the relative density of immunoreactive bands, images were scanned and analysed by the Image J software (National Institute of Health). The experiments were independently performed three times.

Affinity precipitation of activated Ras. For the affinity precipitation of activated Ras, cells were lysed in lysis buffer (50 mM Tris pH 7.5, $10 \mathrm{~mm} \mathrm{MgCl}_{2}, 0.5 \mathrm{M} \mathrm{NaCl}$, and 2\% Igepal). The supernatant was incubated with $10 \mu \mathrm{l}$ of Raf-Ras-binding domain (RBD)-GST beads (Cytoskeleton Inc., Denver, CO, USA), which selectively 
interacted with active GTP-bound Ras. The beads were washed three times with washing buffer ( $25 \mathrm{~mm}$ Tris $\mathrm{pH} 7.5,30 \mathrm{mM} \mathrm{MgCl}_{2}$, $40 \mathrm{~mm} \mathrm{NaCl}$ ) containing $5 \mathrm{~mm} \mathrm{MgCl}_{2}$ and then boiled in SDS sample buffer. Ras associated with Raf-RBD-GST, and total Ras in cell lysates were detected by western blot analysis using anti-Ras antibody. The experiments were independently performed three times.

Collagen gel droplet embedded culture-drug sensitivity test. Collagen gel droplet embedded culture-drug sensitivity test (CD-DST) was performed using a CD-DST kit provided by Nitta Gelatin, Inc. (Osaka, Japan), according to the previously described method (Kobayashi et al, 1997; Takamura et al, 2002). Fresh specimens were suspended in a cell dispersion enzyme solution 'dispersion enzyme cocktail' (EZ) (Nitta Gelatin Inc., Osaka, Japan) and Hanks' balanced saline solution (HBSS) for enzymatic digestion and then incubated in a collagen gel-coated flask. After the elimination of blood cells and dead cells, living cells were collected and incubated in collagen gel droplet embedded culture at $3 \times 10^{2}$ cells per collagen gel droplet ( $30 \mu$ l per drop). Cells were treated with Fluorouracil (5FU; $5 \mu \mathrm{g} \mathrm{ml}^{-1}$ ) (Taj Pharmaceuticals Ltd., India), Oxaliplatin (OXA; $10 \mu \mathrm{g} \mathrm{ml}^{-1}$ ) (Sanofi Aventis Canada Inc., Laval, QC, Canada), Irinotecan (CPT-11; $1 \mu \mathrm{g} \mathrm{ml}^{-1}$ ) (Pfizer Inc., New York, NY, USA), Cisplatin (CDDP; $10 \mu \mathrm{g} \mathrm{ml}^{-1}$ ) (Taj Pharmaceuticals Ltd, Maharashtra, India) and Paclitaxel (PAC; $15 \mu \mathrm{g} \mathrm{ml}^{-1}$ ) (Bristol-Myers Squibb Company, Melbourne, Australia). The physiological concentrations of these drugs are similar to the in vivo drug concentrations. After cells were incubated in serum-free medium to inhibit fibroblast proliferation for $24 \mathrm{~h}$, only the living cells were stained with neutral red stain. The growth rates of control incubations were calculated as the total volume of collagen embedded gel droplets containing living tumour cells on day 7 divided by the total volume of collagen embedded gel droplets containing living tumour cells on day 0 . The in vitro sensitivity was expressed as the percentage $T / C$ ratio, where $T$ was the total volume of the treated group and $C$ was the total volume of the control group.

Cell proliferation detected by MTT assay. Exponentially growing HT-29 cells were seeded at a density of $5 \times 10^{3}$ cells per well in 96-well plates in $200 \mu \mathrm{l}$ of fresh culture medium containing DMEM without phenol red (Sigma-Aldrich) supplemented with 10\% FBS. After a 24 -h incubation at $37^{\circ} \mathrm{C}$ in $5 \% \mathrm{CO}_{2}, 5 \mathrm{FU}\left(4 \mu \mathrm{g} \mathrm{ml}^{-1}\right)$ was added to the culture medium. Cells were incubated at $37^{\circ} \mathrm{C}$ for the next $24 \mathrm{~h}$. Then, cell growth was determined with a 3-(4, 5-dimethylthiazol-2-yl)-2,5-diphenyl tetrazolium bromide (MTT) assay kit (Sigma-Aldrich). Briefly, $10 \mu \mathrm{l}$ of MTT $\left(5 \mathrm{mg} \mathrm{ml}^{-1}\right)$ was added to each well, and then the cells were incubated for an additional $4 \mathrm{~h}$ at $37^{\circ} \mathrm{C}$. Next, the culture fluid was removed and MTT formazan crystals were dissolved in acidified isopropanol. Finally, the absorbance was measured spectrophotometrically with a microplate reader at a wavelength of $570 \mathrm{~nm}$, and background absorbance was measured at $690 \mathrm{~nm}$ and subtracted. All experiments were independently performed three times and each experiment contained triplicates.

Statistical analysis. The correlation between Sdc-1 and MMP-7 serum levels was evaluated with the Spearman Rank Correlation test. Comparisons between groups were made using the Wilcoxonsigned ranks test, Mann-Whitney test, one-way ANOVA for continuous variables, and Pearson test for comparisons of proportions. Survival curves were estimated using the KaplanMeier method, and the differences in survival rates between groups were compared by log-rank test. All data were analysed by SPSS 17.0 software (SPSS Inc., Chicago, IL, USA). A P-value of $<0.05$ was considered as significant for all tests.

\section{RESULTS}

Activated Sdc-1 shedding and increased MMP-7 expression were observed in colorectal cancer patients. Sdc-1 serum levels in colorectal cancer patients were approximately two times higher than in healthy adults (Figure 1A, dot blot), and Sdc-1 expresses on the cellular membrane of colorectal cancer patients was lower than that on the cellular membrane of healthy adults (Figure 1B, immunohistochemistry), but the mRNA expression of Sdc-1 in colon mucosa of patients is similar to healthy adults (Figure 1C, RT-PCR), these suggesting that Sdc-1 shedding was significantly increased in colon cancer patients $(P=0.002)$, and increased Sdc-1 shedding may result in the decrease of Sdc-1 located in colon mucosa of colorectal cancer patients. The mRNA expression of MMP-7 in colon mucosa of patients was higher than in healthy adults (Figure 1D, RT-PCR).

Positive correlation between serum Sdc-1 and MMP-7 levels. Sdc-1 and MMP-7 serum levels in preoperative patients, postoperative patients and healthy adults were analysed. Sdc-1 and MMP-7 serum levels were expressed as median because they did not have a normal distribution. Serum Sdc-1 levels ranged from 132.88 to $876.77 \mathrm{ng} \mathrm{ml}^{-1}$ (median, $434.43 \mathrm{ng} \mathrm{ml}^{-1}$ ), and serum MMP-7 levels ranged from 43.57 to $864.64 \mathrm{ng} \mathrm{ml}^{-1}$ (median, $\left.534.23 \mathrm{ng} \mathrm{ml}^{-1}\right)$. A positive correlation $\left(R^{2}=0.5185\right)$ between serum Sdc-1 and MMP-7 levels was observed (Figure 1E).

Postoperative Sdc-1 and MMP-7 serum levels were lower than preoperative levels, but still higher than those in healthy adults. Serum MMP-7 levels in postoperative patients (median, $332.70 \mathrm{ng} \mathrm{ml}^{-1}$; range, $34.89-745.65 \mathrm{ng} \mathrm{ml}^{-1}$ ) were lower than in preoperative patients (median, $552.38 \mathrm{ng} \mathrm{ml}^{-1}$; range, 153.25$\left.864.64 \mathrm{ng} \mathrm{ml}^{-1}, P=0.014\right)$, but were higher than in healthy adults (median, $160.54 \mathrm{ng} \mathrm{ml}^{-1}$; range, $43.57-334.10 \mathrm{ng} \mathrm{ml}^{-1}, P=0.002$ ). Moreover, a similar pattern was observed for serum Sdc-1 levels. In postoperative patients, serum Sdc-1 levels (median, $344.27 \mathrm{ng} \mathrm{ml}^{-1}$; range, $108.27-745.35 \mathrm{ng} \mathrm{ml}^{-1}$ ) were lower than in preoperative patients (median, $461.55 \mathrm{ng} \mathrm{ml}^{-1}$; range, $\left.132.88-876.77 \mathrm{ng} \mathrm{ml}^{-1}, P=0.009\right)$, but higher than in healthy adults (median, $253.87 \mathrm{ng} \mathrm{ml}^{-1}$; range, $145.52-367.87 \mathrm{ng} \mathrm{ml}^{-1}$, $P=0.043$ ) (Figure 1F).

Patients with high preoperative Sdc-1 serum levels were not sensitive to chemotherapy. Fifty-two patients with colorectal cancer were divided into a low preoperative Sdc-1 level group (26 patients) and a high preoperative Sdc-1 level group (26 patients). A median value of $461.55 \mathrm{ng} \mathrm{ml}^{-1}$ was used as a cutoff. The volume of collagen embedded gel droplets containing living tumour cells obtained from patients with low Sdc-1 serum levels after the addition of 5FU, OXA, CDDP, CPT-11 and PAC was significantly lower than that of patients with high serum Sdc-1 levels $(P<0.01)$. These results indicate that patients with high Sdc1 serum levels demonstrate significantly lower sensitivity to $5 \mathrm{FU}$, CDDP, OXA, CPT-11 and PAC when compared with patients with low Sdc-1 serum levels (Figure 2A and B).

Disease-free survival of patients with high preoperative Sdc-1 serum levels was significantly shorter than that of patients with low preoperative Sdc-1 serum levels. There was no significant difference in clinicopathological parameters between patients with high preoperative Sdc-1 serum levels and patients with low preoperative Sdc-1 serum levels, including age, gender, number of lymph nodes examined, histological grade, lymphovascular invasion, incised margin, colon perforation or obstruction and postoperative adjuvant chemotherapy cycle. However, there was a statistically significant difference in tumour stage and tumour invasion between patients with high and low preoperative Sdc-1 serum levels $(P=0.032$ and $P=0.042$, respectively) (Table 1$)$. 
A

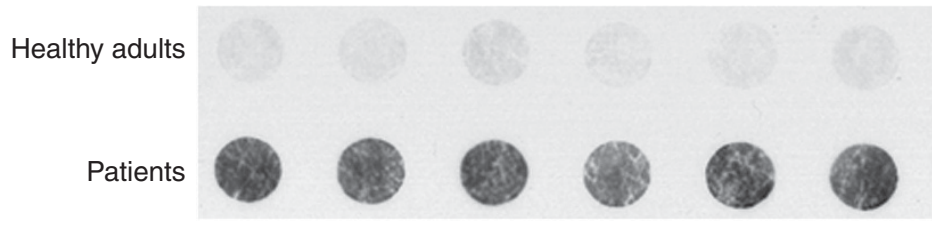

B

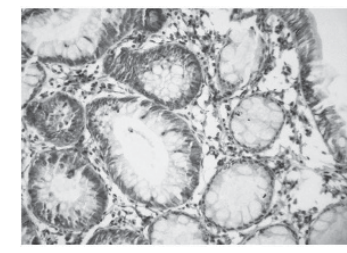

Healthy adults

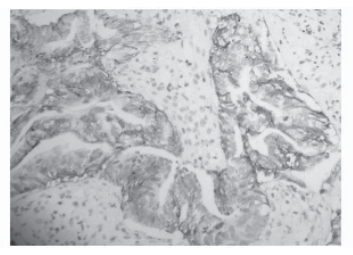

Patients

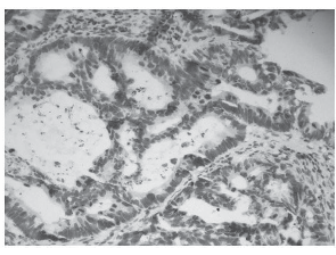

Control

C
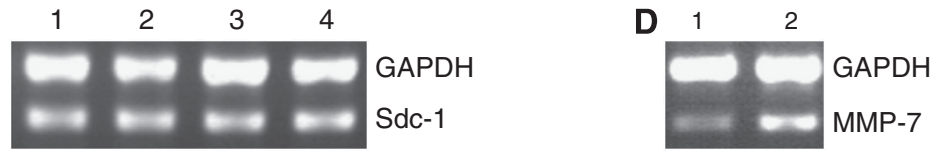

E
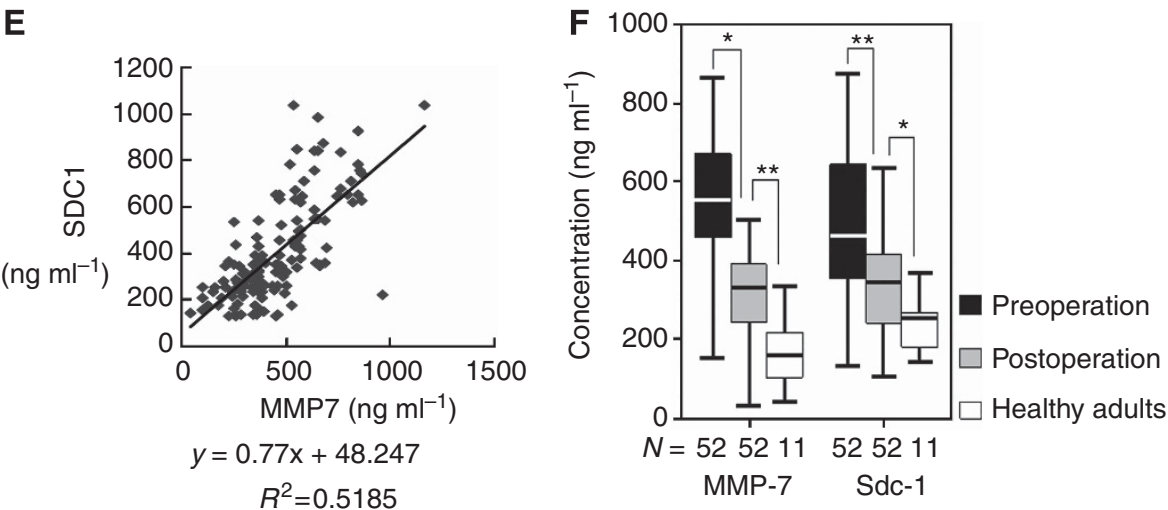

Figure 1. Shed Sdc-1 and MMP-7 levels in serum of colon cancer patients and healthy adults. (A) Sdc-1 shedding was increased in colon cancer patients (dot blotting) $(n=22, P=0.002)$. (B) Sdc-1 expresses on the cellular membrane of colorectal cancer patients and healthy adults (immunohistochemistry) ( $n=44,22$ patients vs 22 healthy adults, $P=0.013)$. (C) The mRNA expression of Sdc- 1 in colon mucosa of patients and healthy adults (RT-PCR) ( $n=44,22$ patients vs 22 healthy adults, 1, 2: healthy adults, 3, 4: patients, $P=0.245)$. (D) The mRNA expression of MMP-7 in colon mucosa of patients and healthy adults (RT-PCR) ( $n=44,22$ patients vs 22 healthy adults, 1 : healthy adults, 2, patients, $P=0.024)$. (E) The positive correlation between Sdc-1 and MMP-7 serum levels (ELISA) ( $n=63$, including preoperative MMP7 and Sdc- 1 serum levels, postoperative MMP7 and Sdc-1 serum levels in 52 patients, and MMP7 and Sdc-1 serum levels in 11 healthy adults) $\left(R^{2}=0.5185\right)$. (F) MMP-7 and Sdc-1 serum levels in postoperative patients $(n=52)$, preoperative patients $(n=52)$ and healthy adults $(n=11)(E L I S A)$. All the values are expressed as the median. ${ }^{*} P \leqslant 0.05$ and ${ }^{* *} P \leqslant 0.01$.

Disease-free survival of patients that underwent colorectal carcinoma resection was compared between the low preoperative Sdc-1 serum level group (26 patients) and the high preoperative Sdc-1 serum level group (26 patients). The disease-free survival rates of patients with low preoperative Sdc-1 serum levels were significantly better than those of patients with high preoperative Sdc-1 serum levels $(P=0.0159$; Figure $2 \mathrm{C})$.

EGFR and KRAS were not mutated in HT-29. In positive controls, EGFR or KRAS mutation was significantly amplified (showing the typical $S$ curve including index phase, linear phase, plateau phase and CT values less than 35). In the HT-29 cell line, EGFR or KRAS mutation showed no amplification (i.e., they did not show the typical S curve), these data indicate that the HT-29 cell line was negative for EGFR or KRAS mutations (Supplementary Figure 1).

MMP-7, MMP-9 or heparanase-induced Sdc-1 shedding enhanced HB-EGF-induced EGFR phosphorylation and induced chemotherapy resistance. It has been reported that activation of EGFR and the downstream Ras-Raf-MEK-ERK pathway is associated with tumour resistance to chemotherapy. Intact HS chains in shed Sdc-1 can bind to HB-EGF and thereby activate EGFR and downstream signalling pathways. To elucidate the mechanism by which shed Sdc-1 enhances chemotherapy resistance in colorectal cancer cells, shed Sdc-1-induced EGFR activation and downstream signal transduction with or without HB-EGF stimulation in HT-29 cells was examined. Addition of MMP-7 significantly decreased Sdc-1 on the cellular membrane (Figure 3A.2) and increased Sdc-1 shedding (Figure 4A.2) and EGFR, MEK, ERK1/2 phosphorylation as well as Ras activation (Figure 4B.2) by 50\%, 486\%, 157\%, 198\%, 96\% and $87 \%$, respectively. In addition, MMP-7 enhanced the proliferation of cells treated with 5FU $(P<0.001$, Figure 4C). Conversely, MMP-7 depletion significantly increased Sdc-1 on the cellular membrane (Figure 3.A3) and inhibited Sdc-1 shedding (Figure 4A.3) and EGFR, MEK, ERK1/2 phosphorylation as well as Ras activation (Figure 4B.3) by $20 \%, 93 \%, 54 \%, 66 \%$, $43 \%$ and $75 \%$, respectively, and reduced the proliferation of cells treated with 5FU $(P<0.001$, Figure 4C). When MMP-7 expression was restored, Sdc-1 on the cellular membrane 
A
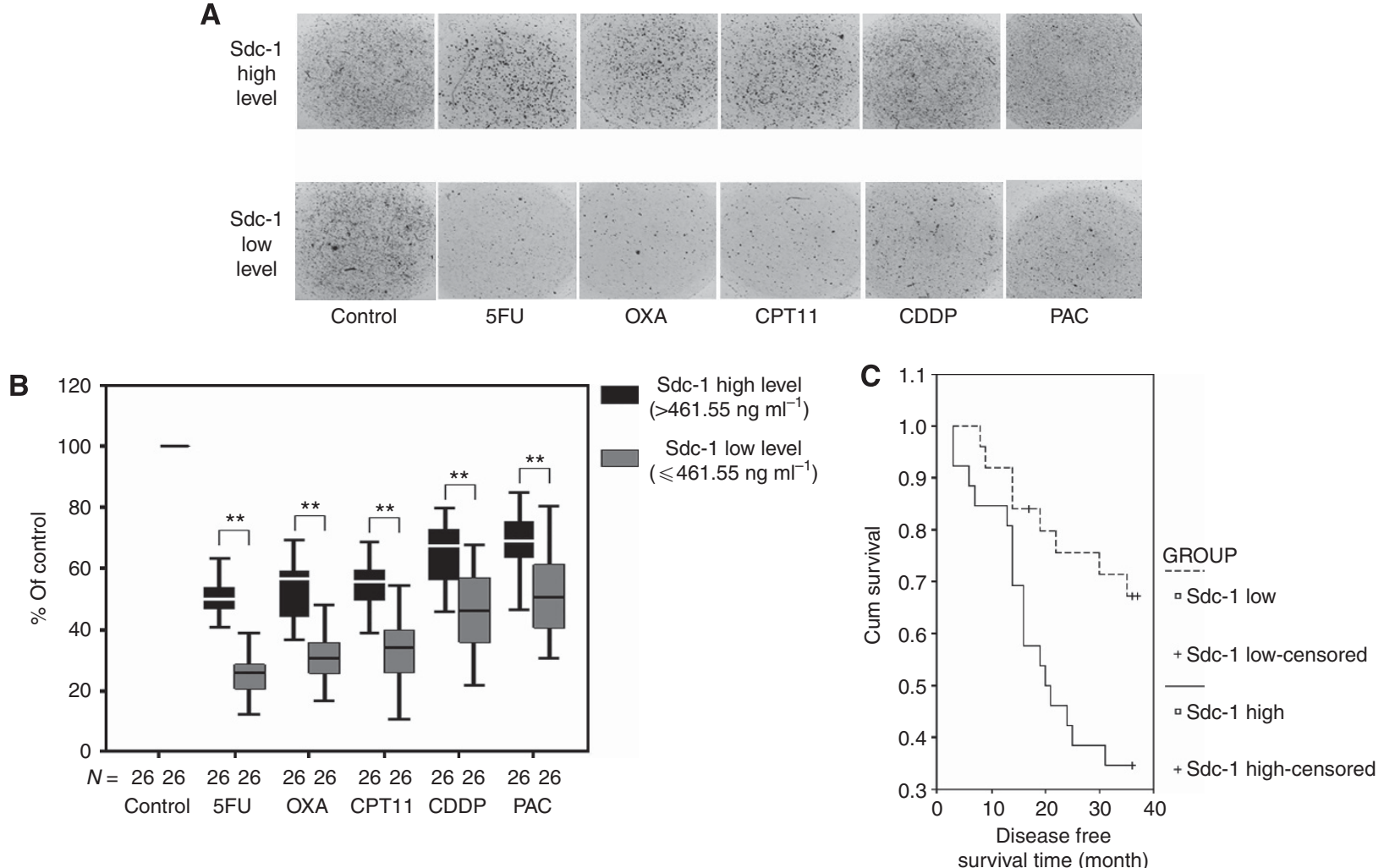

Figure 2. Patients with high Sdc-1 serum levels demonstrated lower sensitivity to 5FU, CDDP, OXA, CPT-11 and PAC treatment than patients with low Sdc-1 serum levels. (A) After the addition of chemotherapeutic drugs, the proliferation of colon cancer cells in patients with high preoperative Sdc-1 serum levels $(n=26)$ was less inhibited than that in patients with low preoperative Sdc-1 serum levels ( $n=26)(C D-D S T)$. (B) Following the addition of chemotherapeutic drugs (at the indicated times), cells were counted and the results were compared with the untreated control cells. All values are expressed as the median. ${ }^{*} P \leqslant 0.01(n=52)$. (C) The disease-free survival time of patients with high preoperative Sdc-1 serum levels $(n=26)$ was significantly shorter than that of patients with low preoperative Sdc-1 serum levels $(n=26)$.

(Figure 3A.4) and Sdc-1 shedding levels (Figure 4A.4) and EGFR, MEK, ERK1/2 phosphorylation as well as Ras activation (Figure 4B.4) returned approximately to levels observed in control cells, and proliferation of cells treated with $5 \mathrm{FU}$ was restored (Figure $4 \mathrm{C}$ ).

The effect of HB-EGF silencing on EGFR phosphorylation and downstream signalling was subsequently examined. Although the addition of MMP7 decreased Sdc-1 level on the cellular membrane (Figure 3A.5) and increased Sdc-1 shedding (Figure 4 A.5) by $62 \%$ and $587 \%$, respectively, HB-EGF depletion significantly inhibited the increase in EGFR, MEK and ERK1/2 phosphorylation and Ras activation (Figure 4B.5) by 19\%, 49\%, $18 \%$ and $36 \%$, respectively, as well as inhibited the proliferation of cells treated with 5FU $(P<0.001$, Figure 4C). Blocking EGFR downstream signal transduction with an EGFR inhibitor, Ras inhibitor, c-Raf inhibitor, MEK inhibitor or ERK inhibitor inhibited the proliferation of cells treated with 5FU $(P<0.001$ respectively, Figure 4D and E).

Meanwhile, addition of heparanase significantly increased MMP-9 level (Figure 5A.3), and addition of MMP-9 or heparanase significantly decreased Sdc-1 on the cellular membrane (Figure 3B.2-3), and increased Sdc-1 shedding (Figure 5A.2-3) and EGFR, MEK, ERK1/2 phosphorylation as well as Ras activation (Figure 5B.2-3). In addition, MMP-9 enhanced the proliferation of cells treated with 5FU $(P<0.001$, Figure 5C). Although the addition of MMP-9 or heparanase decreased Sdc-1 level on the cellular membrane(Figure 3B.4-5) and increased Sdc-1 shedding (Figure 5A.4-5), HB-EGF depletion significantly inhibited the increase in EGFR, MEK and ERK1/2 phosphorylation and
Ras activation (Figure 5B.4-5), as well as inhibited the proliferation of cells treated with 5FU $(P<0.001$, Figure 5C).

These results indicated that shed $\mathrm{Sdc}-1$ activates EGFR phosphorylation and downstream Ras-Raf-MEK-ERK pathways via $\mathrm{HB}-\mathrm{EGF}$ and thereby promotes drug resistance. Collectively, these results suggest that Sdc-1 shedding has an important role in the activation of HB-EGF-induced Ras-Raf-MEK-ERK pathways.

\section{DISCUSSION}

Sdc-1 is mainly expressed on the surface of epithelial and plasma cells, but can exist in the serum as soluble Sdc-1 after proteolytic cleavage by proteases (Brule et al, 2006; Chen et al, 2009; Purushothaman et al, 2010). Shedding of the ectodomain is biologically important, converting the cell-bound Sdc-1 to a soluble active ligand. Sdc-1 shedding is regulated by several known mechanisms. The Sdc-1 ectodomains are cleaved by various secreted and membrane-associated MMPs that include MMP-7, MMP-9, membrane type 1 (MT1)-MMP (MT-MMP1), ADAM10, ADAM17 and a disintegrin (Li et al, 2002; Endo et al, 2003; Brule et al, 2006; Su et al, 2008; Pruessmeyer et al, 2010). They act by proteolytical cleavage of the juxtamembrane site of the core protein. It is also known that FGF-2 activates MMP-7 mediated shedding (Ding et al, 2005) and heparanase accelerates MMP-9 mediated shedding of Sdc-1 (Purushothaman et al, 2010). MMP-9 induction required heparanase enzyme activity and is mediated by the ERK signalling pathway, and urokinase-type plasminogen 

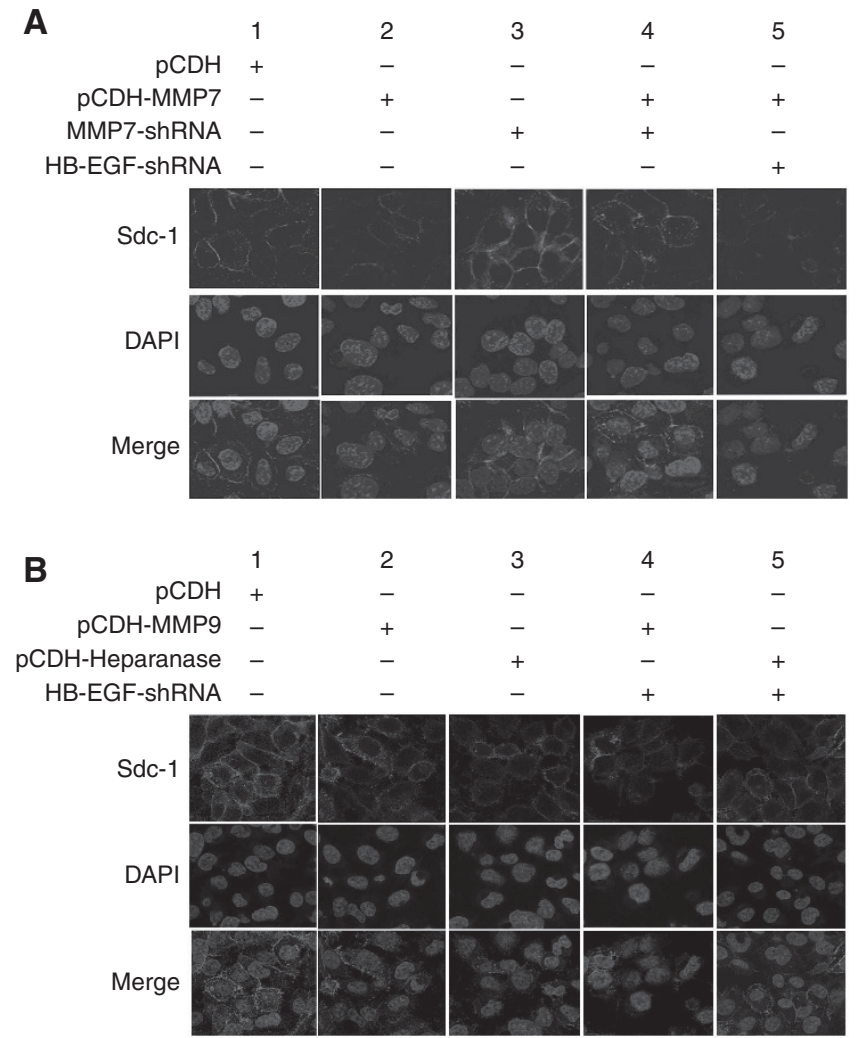

Figure 3. Sdc-1 level on the cellular membrane with MMP7, MMP-9, heparanase and HB-EGF intervention. (A.1) Sdc-1 expressed on the cellular membrane could be detected in colon cancer cells transfected with $\mathrm{pCDH}$ vectors (empty vectors control) by immunofluorescence. (A.2) Decreased Sdc-1 level on the cellular membrane could be detected in colon cancer cells transfected with PCDH-MMP7 vectors (MMP7 overexpression). (A.3) Increased Sdc-1 level on the cellular membrane could be detected in colon cancer cells transfected with MMP7-shRNA (MMP7 silencing). (A.4) Sdc-1 level on the cellular membrane was restored in MMP7-shRNA-treated colon cancer cells following transfection with $\mathrm{PCDH}-\mathrm{MMP7}$ (restoring the expression of MMP7). (A.5) Decreased Sdc-1 level on the cellular membrane could be detected in colon cancer cells transfected with PCDH-MMP7 and HB-EGF-shRNA (MMP7 overexpression and HB-EGF silencing). (B.1) Sdc-1 expressed on the cellular membrane could be detected in colon cancer cells transfected with $\mathrm{pCDH}$ vectors (empty vectors control) by immunofluorescence. (B.2) Decreased Sdc-1 level on the cellular membrane could be detected in colon cancer cells transfected with pCDH-MMP9 vectors (MMP9 overexpression). (B.3) Decreased Sdc-1 level on the cellular membrane could be detected in colon cancer cells transfected with $\mathrm{pCDH}$-heparanase vectors (heparanase overexpression). (B.4) Decreased Sdc-1 level on the cellular membrane could be detected in colon cancer cells transfected with PCDH-MMP9 and HB-EGF-shRNA (MMP9 overexpression and HB-EGF silencing). (B.5) Decreased Sdc-1 level on the cellular membrane could be detected in colon cancer cells transfected with $\mathrm{PCDH}$-heparanase and HB-EGF-shRNA (heparanase overexpression and HB-EGF silencing).

activator (uPA) and its receptor (uPAR), molecular determinants responsible for MMP-9 activation, are upregulated by heparanase (Mahtouk et al, 2007). Moreover, the Sdc-1 itself has an active role in Sdc-1 shedding, as phosphorylation of tyrosine residues present in the cytoplasmic domain (Reiland et al, 1996) and the interaction of Rab5 with the cytoplasmic domain (Hayashida et al, 2008) have been shown to control cleavage of the ectodomain, but not surface expression of Sdc-1. In addition, the GAG chains of Sdc-1 anchor metalloproteinases to the cell surface, thus induce Sdc-1 shedding in epithelial cells (Georges et al, 2012). It was demonstrated that the HS chains on the Sdc-1 core protein suppresses shedding, reducing the amount of HS chains by addition of recombinant human heparanase, can renders Sdc-1 core protein highly susceptible to cleavage by metalloproteases, thus elevates Sdc-1 shedding dramatically (Yang et al, 2007). Recently, it was found that chemotherapy can induce shedding of Sdc-1, particularly via ADAMs and this shed Sdc-1 being functionally active, leads to establishment of a more aggressive phenotype in case of cancer relapse (Ramani and Sanderson, 2014). The process of Sdc-1 shedding is mediated by protein kinase $\mathrm{C}$, protein tyrosine kinase and mitogen-activated protein kinase (MAPK) signal transduction pathways (Wang et al, 2005; Brule et al, 2006; Chen et al, 2009). Rab5 is a critical regulator of Sdc-1 shedding that serves as an on-off molecular switch through its alternation between the GDP-bound and GTP-bound form (Hayashida et al, 2008).

Soluble Sdc-1 is present at low levels in the serum of healthy people, but elevated Sdc-1 levels have been reported in cancer patients (Joensuu et al, 2002; Anttonen et al, 2003, 2006; Yang et al, 2007). In our study, we simultaneously examined Sdc-1 and MMP7 serum levels in colorectal cancer patients and in healthy adults. The results of our study show that Sdc-1 shedding was activated in patients. Moreover, serum Sdc-1 levels in postoperative patients were lower than in preoperative patients, but still higher than those observed in healthy adults. A positive correlation between Sdc-1 and MMP-7 serum expression was observed. Since MMP-7 proteolysis is an important mechanism of Sdc-1 shedding, and MMP-7 can be secreted by colorectal cancer cells and has a pivotal role in almost every stage of colon cancer progression (Kim et al, 2007), we speculate that increased MMP-7 levels lead to enhanced proteolysis of Sdc-1 in colorectal cancer cells, and thus more soluble Sdc-1 is generated. In addition, following colon cancer resection, MMP-7 produced by colorectal cancer cell and MMP-7-induced Sdc-1 shedding was ceased. As MMP7 and Sdc-1 metabolic elimination requires time, serum MMP-7 and Sdc-1 levels do not return to normal values immediately. As expected, in postoperative patients MMP-7 and Sdc-1 levels remained higher than in healthy adults.

It has been reported that high levels of soluble Sdc-1 are associated with tumour growth (Yang et al, 2002; Ding et al, 2005). However, the role of soluble Sdc-1 in chemotherapeutic sensitivity has not been thoroughly examined. Thus, in the present study we examined the impact of Sdc-1 shedding on chemotherapeutic sensitivity of colorectal cancer cells and its possible influence on disease-free survival of colorectal cancer patients. The CD-DST test has been confirmed as a reliable method for measuring chemotherapeutic response (Takamura et al, 2002), which is a newly developed in vitro chemosensitivity test that has several advantages over other conventionally used tests. These advantages include high success rate in primary culture, lower number of required cells $(1 \times 10 \mathrm{E} 5)$, image analysis, which eliminates fibroblast contamination, maintenance of original growth characteristics, and the use of physiological drug concentrations (Kobayashi et al, 2001). Feasibility of CD-DST has been reported in breast, pancreatic, biliary tract and lung cancers, and a direct correlation between CD-DST and chemotherapeutic response has been confirmed. Moreover, high sensitivity and specificity of CDDST have been confirmed, as well as its clinical utility in predicting chemotherapeutic response (Takamura et al, 2002). CD-DST seems to be especially useful in the decision making on which drug should be given to patients who receive neoadjuvant, adjuvant or palliative chemotherapy. In addition, the sensitivity to chemotherapy can be evaluated clinically before its initiation, and continuation or cessation of chemotherapy can be decided depending on the results of CD-DST (Ochiai et al, 2012). Indeed, CD-DST is included in protocols in many medical institutions, including the 

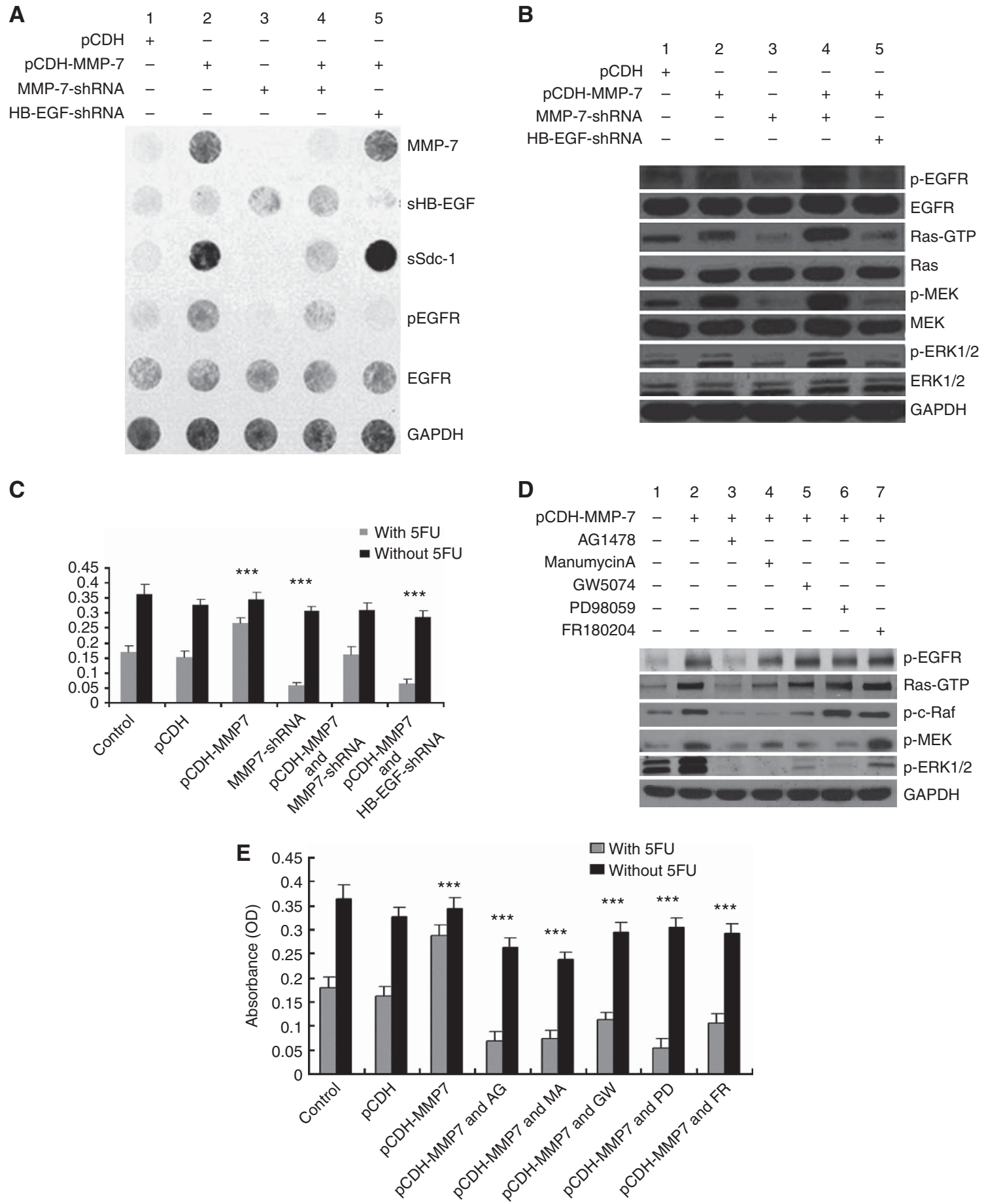

Figure 4. Increased Sdc-1 shedding by MMP7 proteolytic cleavage enhanced EGFR phosphorylation and downstream signal transduction via HB-EGF. Shed Sdc-1 (sSdc-1), soluble HB-EGF (sHB-EGF), MMP-7 in the cell supernatant, and phosphorylated-EGFR (p-EGFR) and EGFR in the cell lysates were detected using dot blotting. (A.1) MMP-7, sSdc-1, sHB-EGF, p-EGFR and EGFR could be detected in colon cancer cells transfected with $\mathrm{pCDH}$ vectors (empty vectors control). (A.2) Increased MMP-7 and sSdc-1 levels and the upregulation of p-EGFR could be detected in colon cancer cells transfected with PCDH-MMP7 vectors (MMP7 overexpression). (A.3) Decreased MMP-7 and sSdc-1 levels and the downregulation of p-EGFR could be detected in colon cancer cells transfected with MMP7-shRNA (MMP7 silencing). (A.4) The expression of MMP-7, sSdc-1 and p-EGFR was restored in MMP7-shRNA-treated colon cancer cells following transfection with PCDH-MMP7 (restoring the expression of MMP7). (A.5) Increased MMP-7 and sSdc-1 levels but not the upregulation of p-EGFR could be detected in colon cancer cells transfected with pCDH-MMP7 and HB-EGF-shRNA (MMP7 overexpression and HB-EGF silencing). (B) p-EGFR, total EGFR, Ras-GTP precipitated with GST-Raf-RBD, total Ras, phosphorylated-MEK (p-MEK), total MEK, phosphorylated-ERK1/2 (p-ERK1/2) and total ERK were detected in cell lysates using western blot analysis. Changes in Ras-GTP, p-MEK and p-ERK1/2 were similar to p-EGFR, but there was no change in total EGFR, total Ras, total MEK and total ERK1/2. (C) After the 24-h incubation with or without 5FU treatment, cell growth in six groups was measured by MTT assay. (D) HT-29 cells were pretreated with the EGFR inhibitor AG1478 $(A G, 1 \mu \mathrm{M})$, the Ras inhibitor manumycin A (MA, $3 \mu \mathrm{M})$, the C-Raf inhibitor GW5074 (GW, $1 \mu \mathrm{M}$ ), the MEK inhibitor PD98059 (PD, $20 \mu \mathrm{M})$ or the ERK1/2 inhibitor FR $180204(F R, 20 \mu \mathrm{M})$, respectively, for $30 \mathrm{~min}$. Cell lysates were prepared and subjected to western blot analysis. (E) Before the 24-h incubation with or without 5FU, HT-29 cells were treated with an EGFR inhibitor, Ras inhibitor, C-Raf inhibitor, MEK inhibitor or ERK1/2 inhibitor and cell growth was measured with an $\mathrm{MTT}$ assay. All values are represented as mean \pm s.d. of triplicate experiments. ${ }^{\star \star \star} P \leqslant 0.001$ vs control in $5 \mathrm{FU}$ group. 
A

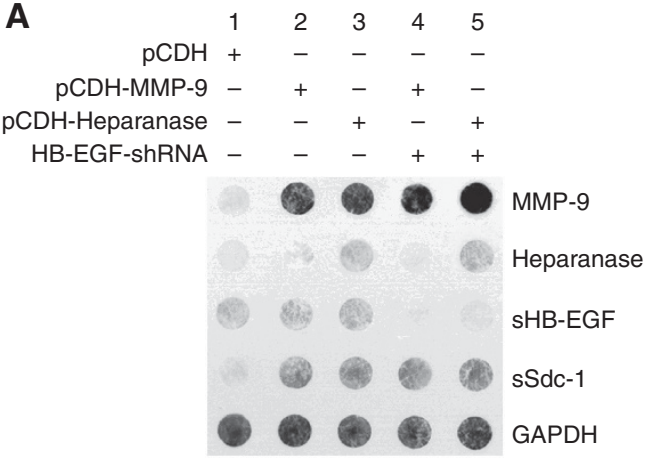

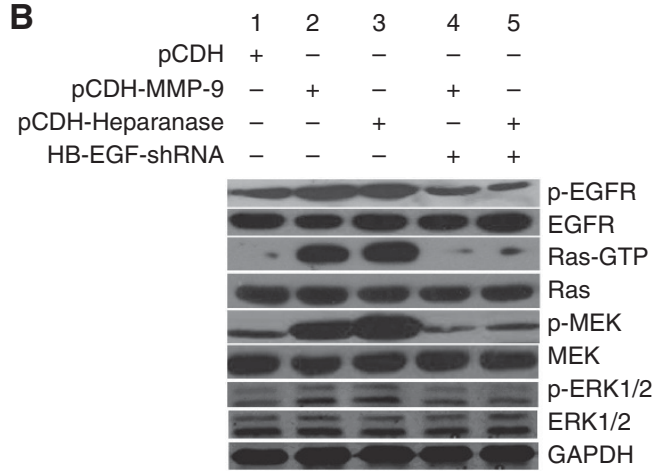

With 5FU

Without $5 \mathrm{FU}$

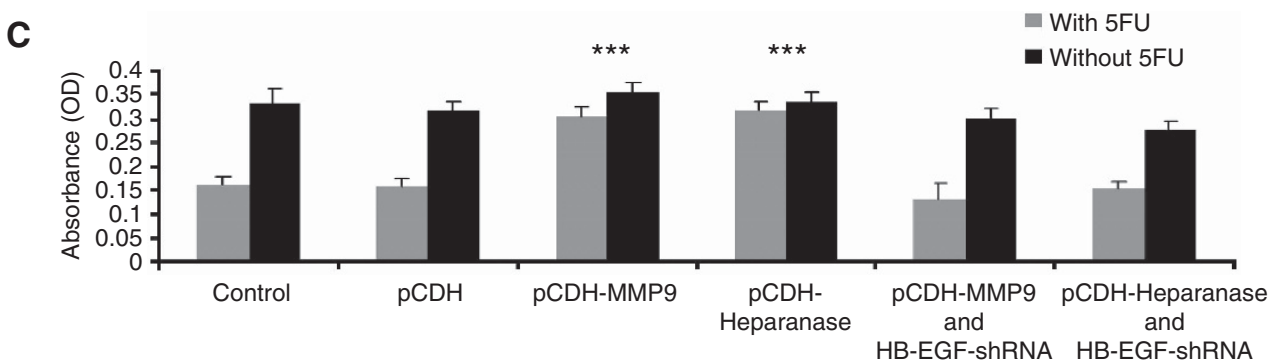

Figure 5. Increased Sdc-1 shedding by MMP9 and heparanase enhanced EGFR phosphorylation and downstream signal transduction via HB-EGF. Shed Sdc-1 (sSdc-1), soluble HB-EGF (sHB-EGF), MMP-9 in the cell supernatant, and heparanase in the cell lysates were detected using dot blotting. p-EGFR, total EGFR, Ras-GTP precipitated with GST-Raf-RBD, total Ras, phosphorylated-MEK (p-MEK), total MEK, phosphorylatedERK1/2 (p-ERK1/2) and total ERK were detected in cell lysates using western blot analysis. (A.1) MMP-9, sSdc-1, sHB-EGF, heparanase and (B.1) p-EGFR, EGFR, Ras-GTP, Ras, p-MEK, MEK and p-ERK1/2, ERK1/2 could be detected in colon cancer cells transfected with pCDH vectors (empty vectors control). (A.2) Increased MMP9 and sSdc-1 levels and (B.2) the upregulation of p-EGFR, Ras-GTP, p-MEK, p-ERK1/2 could be detected in colon cancer cells transfected with $\mathrm{pCDH}-\mathrm{MMP9}$ vectors (MMP9 overexpression). (A.3) Increased MMP9, heparanase and sSdc-1 levels and (B.3) the upregulation of p-EGFR, Ras-GTP, p-MEK, p-ERK1/2 could be detected in colon cancer cells transfected with pCDH-heparanase vectors (heparanase overexpression). (A.4) Increased MMP9, sSdc-1 levels and decreased sHB-EGF could be detected in colon cancer cells transfected with pCDH-MMP9 vectors and HB-EGF-shRNA (MMP9 overexpression and HB-EGF silencing), (B.4) but p-EGFR, Ras-GTP, p-MEK and p-ERK1/2 were downregulated. (A.5) Increased MMP9, heparanase, sSdc-1 levels and decreased sHB-EGF could be detected in colon cancer cells transfected with pCDH-heparanase vectors and HB-EGF-shRNA (heparanase overexpression and HB-EGF silencing), (B.5) but p-EGFR, Ras-GTP, p-MEK and p-ERK1/2 were downregulated. (C) After the 24-h incubation with or without $5 F U$ treatment, cell growth in six groups was measured by MTT assay. ${ }^{\star \star \star} P \leqslant 0.001$ vs control in $5 F U$ group.

Tianjin Medical University Cancer Institute and Hospital of China. We utilised CD-DST in our study to detect chemotherapeutic sensitivity of colorectal cancer patients with different Sdc-1 serum levels. We treated tumour cells isolated from these patients with physiological concentrations of 5FU, OXA, CPT-11, CDDP and PAC. Patients with high preoperative Sdc-1 serum levels demonstrated lower sensitivity to 5FU, OXA, CPT-11, CDDP and PAC than tumour cells obtained from patients with low preoperative Sdc-1 serum levels. As antitumour mechanisms of 5FU, OXA, CPT-11, CDDP and PAC are different, and these patients have not accepted chemotherapy previously, we speculated that serum Sdc-1 may be involved in the primary multidrug resistance (MDR) phenomena. In addition, our study show that there was not a significant difference in clinicopathological parameters, including age, gender, number of lymph nodes examined, histological grade, lymphovascular invasion, incised margin, colon perforation or obstruction and chemotherapy cycle, between patients with high or low preoperative Sdc-1 serum levels. In contrast, the tumour stage was more advanced and tumour invasion was greater in patients with high preoperative Sdc-1 serum levels when compared with patients with low preoperative Sdc-1 serum levels. These results further indicate that soluble Sdc-1 is associated with tumour proliferation and invasion. Meanwhile, the disease-free survival of patients with high preoperative Sdc-1 serum levels was significantly shorter than the disease-free survival of patients with low preoperative Sdc-1 serum levels. This might have been partially due to the difference of preoperative stage, and also might have been due to undetectable residual cancer cells that secreted high levels of soluble Sdc-1, these cancer cells may be insensitive to postoperative adjuvant chemotherapy and lead to cancer recurrence.

Tumour drug resistance involves multiple mechanisms, including p53 mutation, drug-pump induction, and growth-factor related survival and proliferation (McCubrey et al, 2006). Soluble serum Sdc-1, which contains intact HS chains, can bind to numerous growth factors, including HB-EGF (Mythreye and Blobe, 2009). Heparin-binding epithelial growth factor-like factor is synthesised as a single pass membrane-anchored precursor, and the ectodomain of HB-EGF can be proteolytically cleaved by MMP and shed from cell surfaces (Clarke et al, 2006). Notably, the N-terminal sequence in shed HB-EGF is enriched in basic amino acids that are positively charged at physiological $\mathrm{pH}$, enabling interaction with negatively charged heparin sulphate proteoglycans such as Sdc-1 both on the cell surface and in the serum (Higashiyama et al, 1993). Interaction of shed HB-EGF with shed Sdc-1 may enhance EGFR binding affinity to HB-EGF and activate EGFR. In addition, conversion of membrane-bound Sdc-1 into a soluble effector can compete for the same ligands (Mythreye and Blobe, 2009). Activation of EGFR and the downstream RAS-RAF-MEK-ERK signalling pathway accelerate the G1/S-phase transition, G1-arrested cultures showed upregulated chemosensitivity, whereas S phase-arrested cells were more resistant to 
A

Syndecan-1

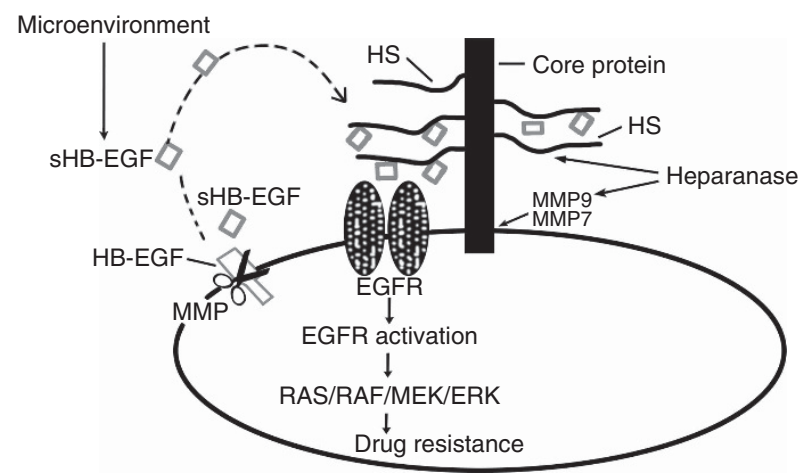

B

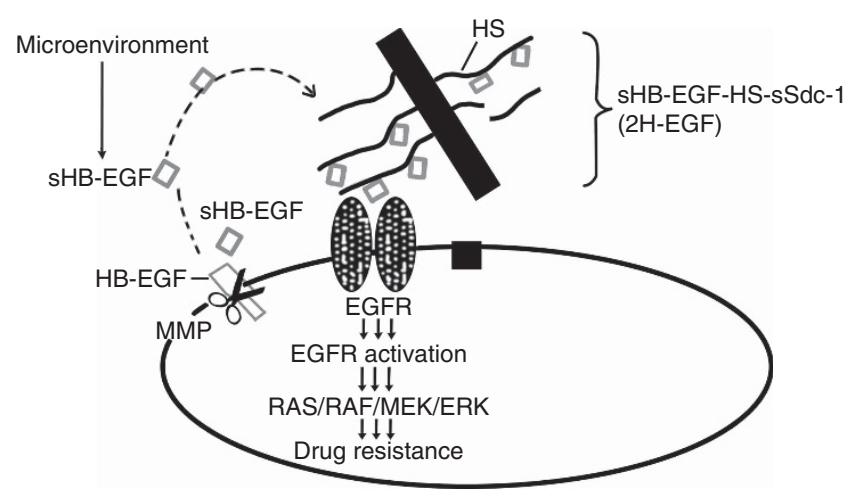

Figure 6. Shed Sdc-1 induces the activation of EGFR and downstream signalling pathways via sHB-EGF. (A) HB-EGF can be proteolytically cleaved by MMP and released as soluble HB-EGF (sHB-EGF). In addition, sHB-EGF can be produced by the cellular microenvironment. sHB-EGF and Sdc-1 form membrane-anchored complexes by binding HS chains situated at the Sdc-1 ectodomain. Together they activate EGFR on adjacent epithelial cells and thereby stimulate the Ras-Raf-MEK-ERK pathway. (B) The Sdc-1 ectodomain can shed from cell surfaces by MMP-7 andMMP-9 proteolysis. Heparanase accelerates shedding of Sdc-1 by inducing MMP-9 expression and reducing the amount of HS chains, thus soluble Sdc-1 (sSdc-1) is formed. In addition, sHB-EGF can bind to HS chains situated at the shed Sdc-1. The structure and function of soluble HB-EGF/Sdc-1 complexes may differ from membrane-anchored HB-EGF/Sdc-1 complexes, and may enhance the activation of EGFR and downstream Ras-Raf-MEK-ERK pathways, thus further promote the resistance to chemotherapy.

chemotherapeutic agents, thus the EGFR-induced G1/S-phase transition can lead to chemotherapeutic agents resistance (Chen et al, 2012). In addition, Sdc- 1 modulates the cancer stem cell phenotype via regulation of the Wnt and IL-6/STAT3 signalling pathways, and the cancer stem cell expresses high levels of MDR proteins, these also demonstrate that Sdc-1 has an important role in MDR (Ibrahim et al, 2013).

On the basis of the above background, we speculate that accelerated Sdc-1 shedding may enhance HB-EGF-induced EGFR and Ras-Raf-MEK-ERK activation. This in turn may interfere with receptors and signal transduction pathways associated with drug metabolism and may have an impact on chemotherapeutic sensitivity of colorectal cancer cells. We found EGFR and KRAS were not mutated in HT-29, thus the research about chemotherapeutical resistance was conducted in HT-29 cell line subsequently. In our study, addition of MMP-7 significantly increased Sdc-1 shedding, and the shed Sdc-1 enhanced the activation of EGFR phosphorylation and downstream Ras-Raf-MEK-ERK pathway, which in turn decreased the chemotherapeutical sensitivity of cancer cells. Meanwhile, MMP-7 depletion significantly inhibited Sdc-1 shedding, thus inhibited the shed Sdc-1-induced EGFR phosphorylation and downstream Ras-Raf-MEK-ERK pathway, which in turn increased the chemotherapeutical sensitivity of cancer cells. When MMP-7 expression was restored, Sdc-1 shedding levels returned approximately to levels observed in control cells; thus, the shed Sdc-1-induced EGFR phosphorylation and downstream Ras-Raf-MEK-ERK pathway returned to normal, and proliferation of cells treated with 5FU was restored. Moreover, these pathways were significantly inhibited and chemotherapeutical sensitivity increased following HB-EGF silencing, even though high levels of shed Sdc-1 caused by MMP-7 were present. These results indicate that MMP-induced accelerated Sdc-1 shedding enhanced the activation of EGFR phosphorylation and downstream Ras-Raf-MEK-ERK pathways via HB-EGF. Furthermore, addition of MMP9 increased Sdc-1 shedding, and addition of heparanase significantly increased MMP9 level, which also significantly increased Sdc-1 shedding, which consistent with previous studies reported (Purushothaman et al, 2010). Another mechanism of heparanase elevating Sdc-1 shedding involves reducing the amount of heparan sulphate chains. There are several potential means by which heparan sulphate chains of Sdc-1 may regulate its shedding. These include physically blocking sheddases from accessing the cleavage sites, stabilising the core protein in a conformation that is less susceptible to proteolysis, and helping to maintain the syndecan-Rab5 complex (Yang et al, 2007). MMP9 and heparanase-induced Sdc-1 shedding enhanced the activation of EGFR phosphorylation and downstream Ras-Raf-MEK-ERK pathway, which in turn decreased the chemotherapeutical sensitivity of cancer cells. But these pathways were significantly inhibited and chemotherapeutical sensitivity increased following HB-EGF silencing, even though high levels of shed Sdc- 1 caused by MMP9 or heparanase were present. These results indicate that not only MMP-7, but also MMP9 or heparanase-induced accelerated Sdc-1 shedding enhanced the activation of EGFR phosphorylation and downstream Ras-Raf-MEK-ERK pathways via HB-EGF. Thus, HB-EGF may have an important role in shed Sdc-1-induced EGFR activation. On the basis of our results, we propose that shed Sdc-1 may enhance the activation of EGFR and downstream signalling pathways via soluble HB-EGF and thereby mediate the resistance to chemotherapy (Figure 6).

In conclusion, accelerated Sdc-1 shedding induced by MMP-7, MMP-9 or heparanase reduced the chemotherapeutic sensitivity of colorectal cancer cells via the activation of HB-EGF and the RasRaf-MEK-ERK pathway. The results of our study underline the importance of Sdc-1 shedding for the development of targeted therapeutic approaches. Therefore, the combined use of Sdc-1 shedding inhibitors, HB-EGF inhibitors and chemotherapy to enhance sensitivity of chemotherapy may be a promising anticancer approach in colorectal cancer. In addition, Sdc-1 serum levels could be a new prognostic marker in colorectal cancer.

\section{ACKNOWLEDGEMENTS}

This work was supported by the grants from the Science Foundation of Tianjin Medical University (No. 2011KY18), National Natural Science Foundation of China (No. 81201946), National Natural Science Foundation of China (No. 81372394), National Science and Technology Major Project (No.2013ZX 09303001) and Specialised Research Fund for the Doctoral Program of Higher Education of China (No. 2012202120013). The funding sponsors had no role in the study design, collection, analysis or interpretation of data, and writing the report. We thank the Department of Breast Pathology at the Tianjin Medical 
University Cancer Institute and Hospital for collagen gel droplet embedded culture-drug sensitivity test (CD-DST).

\section{REFERENCES}

Andrian E, Grenier D, Rouabhia M (2006) Porphyromonas gingivalis gingipains mediate the shedding of syndecan-1 from the surface of gingival epithelial cells. Oral Microbiol Immunol 21: 123-128.

Anegawa G, Kawanaka H, Yoshida D, Konishi K, Yamaguchi S, Kinjo N, Taketomi A, Hashizume M, Shimokawa H, Maehara Y (2008) Defective endothelial nitric oxide synthase signaling is mediated by rho-kinase activation in rats with secondary biliary cirrhosis. Hepatology 47: 966-977.

Anttonen A, Leppa S, Heikkila P, Grenman R, Joensuu H (2006) Effect of treatment of larynx and hypopharynx carcinomas on serum syndecan-1 concentrations. J Cancer Res Clin Oncol 132: 451-457.

Anttonen A, Leppa S, Ruotsalainen T, Alfthan H, Mattson K, Joensuu H (2003) Pretreatment serum syndecan-1 levels and outcome in small cell lung cancer patients treated with platinum-based chemotherapy. Lung Cancer 41: 171-177.

Brule S, Charnaux N, Sutton A, Ledoux D, Chaigneau T, Saffar L, Gattegno L (2006) The shedding of syndecan-4 and syndecan- 1 from HeLa cells and human primary macrophages is accelerated by SDF-1/CXCL12 and mediated by the matrix metalloproteinase-9. Glycobiology 16: 488-501.

Chen P, Abacherli LE, Nadler ST, Wang Y, Li Q, Parks WC (2009) MMP7 shedding of syndecan-1 facilitates re-epithelialization by affecting alpha(2)beta(1) integrin activation. PLoS ONE 4: e6565.

Chen SJ, Luan J, Zhang HS, Ruan CP, Xu XY, Li QQ, Wang NH (2012) EGFR-mediated G1/S transition contributes to the multidrug resistance in breast cancer cells. Mol Biol Rep 39: 5465-5471.

Clarke PA, Dickson JH, Harris JC, Grabowska A, Watson SA (2006) Gastrin enhances the angiogenic potential of endothelial cells via modulation of heparin-binding epidermal-like growth factor. Cancer Res 66: 3504-3512.

Ding K, Lopez-Burks M, Sanchez-Duran JA, Korc M, Lander AD (2005) Growth factor-induced shedding of syndecan- 1 confers glypican-1 dependence on mitogenic responses of cancer cells. J Cell Biol 171: 729-738.

Endo K, Takino T, Miyamori H, Kinsen H, Yoshizaki T, Furukawa M, Sato H (2003) Cleavage of syndecan-1 by membrane type matrix metalloproteinase-1 stimulates cell migration. J Biol Chem 278: 40764-40770.

Fitzgerald ML, Wang Z, Park PW, Murphy G, Bernfield M (2000) Shedding of syndecan- 1 and -4 ectodomains is regulated by multiple signaling pathways and mediated by a TIMP-3-sensitive metalloproteinase. J Cell Biol 148: 811-824.

Fujiya M, Watari J, Ashida T, Honda M, Tanabe H, Fujiki T, Saitoh Y, Kohgo Y (2001) Reduced expression of syndecan-1 affects metastatic potential and clinical outcome in patients with colorectal cancer. Jpn J Cancer Res 92: 1074-1081.

Georges S, Heymann D, Padrines M (2012) Modulatory effects of proteoglycans on proteinase activities. Methods Mol Biol 836: 307-322.

Gotte M (2003) Syndecans in inflammation. FASEB J 17: 575-591.

Hashimoto Y, Skacel M, Adams JC (2008) Association of loss of epithelial syndecan-1 with stage and local metastasis of colorectal adenocarcinomas: an immunohistochemical study of clinically annotated tumors. $B M C$ Cancer 8: 185.

Hayashida K, Stahl PD, Park PW (2008) Syndecan-1 ectodomain shedding is regulated by the small GTPase Rab5. J Biol Chem 283: 35435-35444.

Higashiyama S, Abraham JA, Klagsbrun M (1993) Heparin-binding EGF-like growth factor stimulation of smooth muscle cell migration: dependence on interactions with cell surface heparan sulfate. J Cell Biol 122: 933-940.

Ibrahim SA, Hassan H, Vilardo L, Kumar SK, Kumar AV, Kelsch R, Schneider C, Kiesel L, Eich HT, Zucchi I, Reinbold R, Greve B, Gotte M (2013) Syndecan-1 (CD138) modulates triple-negative breast cancer stem cell properties via regulation of LRP-6 and IL-6-mediated STAT3 signaling. PLOS ONE 8: e85737.

Joensuu H, Anttonen A, Eriksson M, Makitaro R, Alfthan H, Kinnula V, Leppa S (2002) Soluble syndecan-1 and serum basic fibroblast growth factor are new prognostic factors in lung cancer. Cancer Res 62: 5210-5217.

Kim M, Murakami A, Ohigashi H (2007) Modifying effects of dietary factors on (-)-epigallocatechin-3-gallate-induced pro-matrix metalloproteinase-7 production in HT-29 human colorectal cancer cells. Biosci Biotechnol Biochem 71: 2442-2450.

Kobayashi H, Higashiyama M, Minamigawa K, Tanisaka K, Takano T, Yokouchi H, Kodama K, Hata T (2001) Examination of in vitro chemosensitivity test using collagen gel droplet culture method with colorimetric endpoint quantification. Jpn J Cancer Res 92: 203-210.

Kobayashi H, Tanisaka K, Doi O, Kodama K, Higashiyama M, Nakagawa H, Miyake M, Taki T, Hara S, Yasutomi M, Hanatani Y, Kotake K, Kubota T (1997) An in vitro chemosensitivity test for solid human tumors using collagen gel droplet embedded cultures. Int J Oncol 11: 449-455.

Li Q, Park PW, Wilson CL, Parks WC (2002) Matrilysin shedding of syndecan-1 regulates chemokine mobilization and transepithelial efflux of neutrophils in acute lung injury. Cell 111: 635-646.

Mahtouk K, Hose D, Raynaud P, Hundemer M, Jourdan M, Jourdan E, Pantesco V, Baudard M, De Vos J, Larroque M, Moehler T, Rossi JF, Reme T, Goldschmidt H, Klein B (2007) Heparanase influences expression and shedding of syndecan-1, and its expression by the bone marrow environment is a bad prognostic factor in multiple myeloma. Blood 109: 4914-4923.

McCubrey JA, Steelman LS, Abrams SL, Lee JT, Chang F, Bertrand FE, Navolanic PM, Terrian DM, Franklin RA, D'Assoro AB, Salisbury JL, Mazzarino MC, Stivala F, Libra M (2006) Roles of the RAF/MEK/ERK and PI3K/PTEN/AKT pathways in malignant transformation and drug resistance. Adv Enzyme Regul 46: 249-279.

Mennerich D, Vogel A, Klaman I, Dahl E, Lichtner RB, Rosenthal A, Pohlenz HD, Thierauch KH, Sommer A (2004) Shift of syndecan-1 expression from epithelial to stromal cells during progression of solid tumours. Eur J Cancer 40: 1373-1382.

Mythreye K, Blobe GC (2009) Proteoglycan signaling co-receptors: roles in cell adhesion, migration and invasion. Cell Signal 21: 1548-1558.

Nikolova V, Koo CY, Ibrahim SA, Wang Z, Spillmann D, Dreier R, Kelsch R, Fischgrabe J, Smollich M, Rossi LH, Sibrowski W, Wulfing P, Kiesel L, Yip GW, Gotte M (2009) Differential roles for membrane-bound and soluble syndecan-1 (CD138) in breast cancer progression. Carcinogenesis 30: 397-407.

Ochiai T, Nishimura K, Watanabe T, Kitajima M, Nakatani A, Inou T, Washio M, Sakuyama N, Sato T, Kishine K, Ochi T, Okubo S, Futagawa S, Mashiko S, Nagaoka I (2012) Individualized chemotherapy for colorectal cancer based on the collagen gel droplet-embedded drug sensitivity test. Oncol Lett 4: 621-624.

Pentheroudakis G, Kotoula V, De Roock W, Kouvatseas G, Papakostas P, Makatsoris T, Papamichael D, Xanthakis I, Sgouros J, Televantou D, Kafiri G, Tsamandas AC, Razis E, Galani E, Bafaloukos D, Efstratiou I, Bompolaki I, Pectasides D, Pavlidis N, Tejpar S, Fountzilas G (2013) Biomarkers of benefit from cetuximab-based therapy in metastatic colorectal cancer: interaction of EGFR ligand expression with RAS/RAF, PIK3CA genotypes. BMC Cancer 13: 49.

Pruessmeyer J, Martin C, Hess FM, Schwarz N, Schmidt S, Kogel T, Hoettecke N, Schmidt B, Sechi A, Uhlig S, Ludwig A (2010) A disintegrin and metalloproteinase 17 (ADAM17) mediates inflammation-induced shedding of syndecan-1 and -4 by lung epithelial cells. J Biol Chem 285: 555-564.

Purushothaman A, Uyama T, Kobayashi F, Yamada S, Sugahara K, Rapraeger AC, Sanderson RD (2010) Heparanase-enhanced shedding of syndecan-1 by myeloma cells promotes endothelial invasion and angiogenesis. Blood 115: 2449-2457.

Ramani VC, Sanderson RD (2014) Chemotherapy stimulates syndecan-1 shedding: a potentially negative effect of treatment that may promote tumor relapse. Matrix Biol 35: 215-222.

Reiland J, Ott VL, Lebakken CS, Yeaman C, McCarthy J, Rapraeger AC (1996) Pervanadate activation of intracellular kinases leads to tyrosine phosphorylation and shedding of syndecan-1. Biochem J 319(Pt 1): 39-47.

Su G, Blaine SA, Qiao D, Friedl A (2008) Membrane type 1 matrix metalloproteinase-mediated stromal syndecan-1 shedding stimulates breast carcinoma cell proliferation. Cancer Res 68: 9558-9565.

Takamura Y, Kobayashi H, Taguchi T, Motomura K, Inaji H, Noguchi S (2002) Prediction of chemotherapeutic response by collagen gel droplet embedded culture-drug sensitivity test in human breast cancers. Int $J$ Cancer 98: 450-455.

Teng YH, Aquino RS, Park PW (2012) Molecular functions of syndecan-1 in disease. Matrix Biol 31: 3-16.

Wang X, Chen Y, Song Y, Zhang S, Xie X (2011) Activated Syndecan-1 shedding contributes to mice colitis induced by dextran sulfate sodium. Dig Dis Sci 56: 1047-1056. 
Wang Z, Gotte M, Bernfield M, Reizes O (2005) Constitutive and accelerated shedding of murine syndecan-1 is mediated by cleavage of its core protein at a specific juxtamembrane site. Biochemistry 44: $12355-12361$.

Yang Y, Macleod V, Miao HQ, Theus A, Zhan F, Shaughnessy Jr. JD, Sawyer J, Li JP, Zcharia E, Vlodavsky I, Sanderson RD (2007) Heparanase enhances syndecan-1 shedding: a novel mechanism for stimulation of tumor growth and metastasis. J Biol Chem 282: 13326-13333.

Yang Y, Yaccoby S, Liu W, Langford JK, Pumphrey CY, Theus A, Epstein J, Sanderson RD (2002) Soluble syndecan-1 promotes growth of myeloma tumors in vivo. Blood 100: 610-617.
Zhang S, Qing Q, Wang Q, Xu J, Zhi F, Park PW, Zhang Y, Chen Y (2013) Syndecan-1 and heparanase: potential markers for activity evaluation and differential diagnosis of Crohn's disease. Inflamm Bowel Dis 19: $1025-1033$.

This work is published under the standard license to publish agreement. After 12 months the work will become freely available and the license terms will switch to a Creative Commons AttributionNonCommercial-Share Alike 3.0 Unported License.

Supplementary Information accompanies this paper on British Journal of Cancer website (http://www.nature.com/bjc) 